\title{
Transient analysis of a stationary Lévy-driven queue
}

\author{
Jevgenijs Ivanovs ${ }^{\dagger}$ and Michel Mandjes ${ }^{\bullet} \star \star$
}

September 14, 2021

\begin{abstract}
In this paper we study a queue with Lévy input, without imposing any a priori assumption on the jumps being one-sided. The focus is on computing the transforms of all sorts of quantities related to the transient workload, assuming the workload is in stationarity at time 0 . The results are simple expressions that are in terms of the bivariate Laplace exponents of ladder processes. In particular, we derive the transform of the minimum workload attained over an exponentially distributed interval.
\end{abstract}

Keywords. Queues $\star$ Lévy processes $\star$ conditioned to stay positive

† University of Lausanne, Quartier UNIL-Dorigny Bâtiment Extranef, 1015 Lausanne, Switzerland.

- Korteweg-de Vries Institute for Mathematics, University of Amsterdam, Science Park 904, 1098 XH Amsterdam, the Netherlands.

* CWI, P.O. Box 94079, 1090 GB Amsterdam, the Netherlands.

J. Ivanovs is supported by the Swiss National Science Foundation Project 200020_143889.

M. Mandjes is also with Eurandom, Eindhoven University of Technology, Eindhoven, the Netherlands, and IBIS, Faculty of Economics and Business, University of Amsterdam, Amsterdam, the Netherlands. M. Mandjes' research is partly funded by the NWO Gravitation project NETWORKS, grant number 024.002.003.

\section{Introduction}

In this short communication we focus on the analysis of various random quantities related to the transient of a Lévy-driven queue. Throughout, we denote the driving Lévy process by $X_{t}, t \geq 0$, uniquely characterized through its Lévy exponent

$$
\psi(\theta)=\log \mathbb{E} e^{\mathrm{i} \theta X_{1}}, \quad \theta \in \mathbb{R} .
$$

As usual, the corresponding queueing process (or: workload process) $Q_{t}, t \geq 0$ is defined as the solution to the Skorokhod problem [5, Ch. II], i.e.,

$$
Q_{t}=Q_{0}+X_{t}-\left(\underline{X}_{t}+Q_{0}\right) \wedge 0=Q_{0} \vee\left(-\underline{X}_{t}\right)+X_{t}, \quad t \geq 0,
$$

where $\underline{X}_{t}:=\inf _{s \in[0, t]} X_{s}$ is the running minimum and $Q_{0}$ is assumed to be independent of the evolution of the driving process $X_{t}$ for $t \geq 0$; similarly we let $\bar{X}_{t}:=\sup _{s \in[0, t]} X_{s}$.

It is well known that $Q_{t}$ converges to a stationary distribution as $t \rightarrow \infty$ if and only if $X_{t}$ has a negative drift, that is, $\lim _{t \rightarrow \infty} X_{t}=-\infty$ a.s.; we therefore assume that this condition is in place throughout this work. If $\mathbb{E} X_{1}$ is well-defined, then this condition is equivalent to requiring that $\mathbb{E} X_{1} \in[-\infty, 0)$, see [6, Thm. 7.2]. It is also a standard result that the stationary distribution of $Q_{t}$ coincides with the 
distribution of $\bar{X}:=\bar{X}_{\infty}$, the overall supremum; this is a property that is often attributed to Reich [5, Eqn. (2.5)].

Importantly, in the sequel we systematically assume that $Q_{0}$ has the stationary distribution; as will become clear, this assumption plays a crucial role in the analysis. As mentioned above, the primary objective of this note is to study various transient metrics related to the process $Q_{t}$. All our results are in terms of transforms of the quantities of interest, in addition transformed with respect to time. In this respect, it is recalled that taking transforms with respect to time essentially amounts to considering $t=e_{q}$, where $e_{q}$ is an exponential variable with rate $q$ (i.e., with mean $q^{-1}$ ), sampled independently of everything else.

This note is organized as follows. In Section 2 we sketch preliminaries, related to splitting at extrema and the Wiener-Hopf factorization. In Section 3 we focus on the distribution of the minimum workload attained over an exponentially distributed amount of time. Section 4 provides transforms of some other quantities of interest, distinguishing between two cases: (i) the ongoing busy period and (ii) the finished initial busy period, in which case the focus is on the so-called unused capacity. Finally, Section 5 focuses on the minimal workload in a queue conditioned to be positive. We conclude the paper by mentioning some challenging open problems.

\section{Preliminaries}

Let us state some well-known facts about splitting and the Wiener-Hopf factorization, all of which can be found in e.g. $[1, \mathrm{Ch} . \mathrm{VI}]$. Consider the process $X$ on the interval $\left[0, e_{q}\right]$ and let

$$
\underline{G}_{e_{q}}:=\sup \left\{t \in\left[0, e_{q}\right]: X_{t} \wedge X_{t-}=\underline{X}_{e_{q}}\right\}
$$

be the (last) time of the minimum. It is well known that the process $X$ splits at its minimum, see $[1$, Lem. VI.6]. This result can be stated in a simple form when considering the process $X_{t}^{\prime}:=X_{t}$ for all $t \neq \underline{G}_{e_{q}}$ and $X_{\underline{G}_{e_{q}}}^{\prime}:=\underline{X}_{e_{q}}$. Namely, the processes

$$
\left\{X_{t}^{\prime}, t \in\left[0, \underline{G}_{e_{q}}\right]\right\} \text { and }\left\{X_{t+\underline{G}_{e_{q}}}^{\prime}-X_{\underline{G}_{e_{q}}}^{\prime}, t \in\left[0, e_{q}-\underline{G}_{e_{q}}\right]\right\}
$$

are independent (note that $X^{\prime}$ and $X$ differ only if $X$ jumps up at $\underline{G}_{e_{q}}$ - this jump should be included in the right process, but not in the left). In particular, the above implies that $\underline{X}_{e_{q}}$ and $X_{e_{q}}-\underline{X}_{e_{q}}$ are independent. Splitting in the queueing context used earlier; see e.g. [3].

Let us define the following two functions for $\alpha, \beta \in \mathbb{C}$ with $\Re(\alpha), \Re(\beta) \geq 0$ :

$$
\begin{aligned}
& \bar{\kappa}(\alpha, \beta)=\exp \left(\int_{0}^{\infty} \int_{(0, \infty)}\left(e^{-t}-e^{-\alpha t-\beta x}\right) \frac{1}{t} \mathbb{P}\left(X_{t} \in \mathrm{d} x\right) \mathrm{d} t\right), \\
& \underline{\kappa}(\alpha, \beta)=\exp \left(\int_{0}^{\infty} \int_{(-\infty, 0]}\left(e^{-t}-e^{-\alpha t-\beta x}\right) \frac{1}{t} \mathbb{P}\left(X_{t} \in \mathrm{d} x\right) \mathrm{d} t\right),
\end{aligned}
$$

which are the Laplace exponents of the so-called (strictly) ascending and (weakly) descending bivariate ladder processes, respectively. In general these functions are defined up to a multiplicative constant; the trivial scaling chosen in (2) implies that

$$
\bar{\kappa}(\alpha, 0) \underline{\kappa}(\alpha, 0)=\exp \left(\int_{0}^{\infty} \int_{(0, \infty)}\left(e^{-t}-e^{-\alpha t}\right) \frac{1}{t} \mathrm{~d} t\right)=\alpha, \quad \alpha>0,
$$


by virtue of the Frullani integral identity. This particular scaling simplifies the calculations in the proofs, but it does not affect the results of the paper. The Laplace exponents can then be used to express the so-called Wiener-Hopf factors:

$$
\begin{aligned}
\mathbb{E} e^{-\alpha \underline{G}_{e_{q}}+\beta \underline{X}_{e_{q}}}=\frac{\underline{\kappa}(q, 0)}{\underline{\kappa}(q+\alpha, \beta)}, \\
\mathbb{E} e^{-\alpha\left(e_{q}-\underline{G}_{e_{q}}\right)-\beta\left(X_{e_{q}}-\underline{X}_{e_{q}}\right)}=\mathbb{E} e^{-\alpha \bar{G}_{e_{q}}-\beta \bar{X}_{e_{q}}}=\frac{\bar{\kappa}(q, 0)}{\bar{\kappa}(q+\alpha, \beta)},
\end{aligned}
$$

where $\bar{G}_{e_{q}}:=\inf \left\{t \in\left[0, e_{q}\right]: X_{t} \vee X_{t-}=\bar{X}_{e_{q}}\right\}$ is the (first) time of the maximum. Finally, for $\beta \in \mathbb{R}$ we have

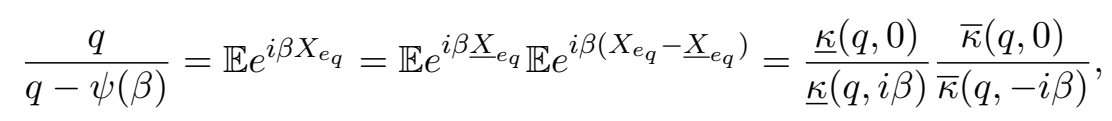

which according to $(3)$ yields

$$
q-\psi(\beta)=\underline{\kappa}(q, i \beta) \bar{\kappa}(q,-i \beta) .
$$

Let us finally remark that one has to distinguish between first and last extrema only in the case when $X$ is a compound Poisson process; the same is true also for open and closed integration sets in (2).

\section{Minimum workload}

In [4] the distribution of the minimum workload during $\left[0, e_{q}\right)$ was characterized for the case the driving Lévy process is spectrally one-sided; it was assumed that the workload is in stationarity at time 0 . In this section, this result is extended to the spectrally two-sided case. Several ramifications of this result are presented as well.

Throughout we let $\underline{Q}_{t}$ be the running minimum of the workload, i.e., $\underline{Q}_{t}$ is the minimum of $Q_{s}$ over $s \in[0, t]$, and we assume that $Q_{0}$ has the stationary workload distribution.

\subsection{Transform of minimum workload}

This subsection shows how to express the transform of $\underline{Q}_{t}$ in terms of the Wiener-Hopf factors. Observe that

$$
\underline{Q}_{t}=\left(Q_{0}+\underline{X}_{t}\right) \vee 0=\underline{X}_{t}+Q_{0} \vee\left(-\underline{X}_{t}\right)
$$

which follows by considering the event $Q_{0}>-\underline{X}_{t}$ and its complement separately. To this end, we first rewrite the definition of $Q_{t}$, as given in (1), in terms of $X_{t}-\underline{X}_{t}$ and $\underline{Q}_{t}$ :

$$
Q_{t}=\left(X_{t}-\underline{X}_{t}\right)+\underline{X}_{t}+Q_{0} \vee\left(-\underline{X}_{t}\right)=\left(X_{t}-\underline{X}_{t}\right)+\underline{Q}_{t},
$$

which is also easily seen from Figure 1. This means that we in particular have

$$
Q_{e_{q}}=\left(X_{e_{q}}-\underline{X}_{e_{q}}\right)+\underline{Q}_{e_{q}},
$$

where the two terms on the right are independent, because of (7) and splitting at the infimum, i.e. $X_{e_{q}}-\underline{X}_{e_{q}}$ and $\underline{X}_{e_{q}}$ are independent, see Section 2. Using (5) we thus obtain the identity

$$
e^{-\theta Q_{e_{q}}}=\mathbb{E} e^{-\theta\left(X_{e_{q}}-\underline{X}_{e_{q}}\right)} \mathbb{E} e^{-\theta \underline{Q}_{e_{q}}}=\mathbb{E} e^{-\theta \bar{X}_{e_{q}}} \mathbb{E} e^{-\theta \underline{Q}_{e_{q}}}=\frac{\bar{\kappa}(q, 0)}{\bar{\kappa}(q, \theta)} \mathbb{E} e^{-\theta \underline{Q}_{e_{q}}}
$$

for $\theta \geq 0$. Since $Q_{0}$ has the stationary workload distribution, so does $Q_{e_{q}}$. As a consequence, $Q_{e_{q}}$ has the distribution of $\bar{X}$, which yields the following result, see also (5). 
Proposition 1. For any $\theta, q \geq 0$,

$$
\mathbb{E} e^{-\theta \underline{Q}_{e}}=\frac{\bar{\kappa}(0,0)}{\bar{\kappa}(0, \theta)} \frac{\bar{\kappa}(q, \theta)}{\bar{\kappa}(q, 0)}
$$

It is noted that Equation (9) generalizes the findings of [4] which just cover the spectrally one-sided cases; it is readily verified that plugging in expressions for $\bar{\kappa}$ for the one-sided cases the formulas obtained are in accordance with those derived in [4].

Remark 1. The transform of $\underline{Q}_{e_{q}}$ has a simple explicit form also in the case when $Q_{0}$ is exponential (with mean $\left.\lambda^{-1}\right)$ :

$$
\begin{aligned}
\mathbb{E} e^{-\theta \underline{Q}_{e_{q}}} & =\mathbb{P}\left(\underline{Q}_{e_{q}}<e_{\theta}\right)=\mathbb{P}\left(Q_{0}+\underline{X}_{e_{q}}<e_{\theta}\right)=\mathbb{P}\left(Q_{0}<e_{\theta}-\underline{X}_{e_{q}}\right)=1-\mathbb{E} e^{-\lambda\left(e_{\theta}-\underline{X}_{e_{q}}\right)} \\
& =1-\frac{\theta}{\lambda+\theta} \frac{\underline{\kappa}(q, 0)}{\underline{\kappa}(q, \lambda)}
\end{aligned}
$$

and then (8) yields $\mathbb{E} e^{-\theta Q_{e_{q}}}$. Throughout this work we assume that $Q_{0}$ has the stationary distribution, but virtually all results carry over to the case of the exponential initial distribution.

We conclude this section by deriving a related, elegant identity. To this end, we define by

$$
\tau:=\inf \left\{t \geq 0: Q_{t} \wedge Q_{t-}=0\right\}=\inf \left\{t \geq 0: Q_{t}=0\right\} \text { a.s., }
$$

the time when the system becomes empty for the first time; $\tau$ is also referred to as the residual busy period as seen from time 0 , and the equality follows from [1, Prop. VI.4]. Clearly, $\mathbb{P}\left(\tau=e_{q}\right)=0$ and as a consequence

$$
\mathbb{P}\left(\underline{Q}_{e_{q}}=0\right)=\mathbb{P}\left(\tau<e_{q}\right) .
$$

Taking $\theta \rightarrow \infty$ in (9), and noting that $\bar{\kappa}(q, \theta) / \bar{\kappa}(0, \theta) \rightarrow 1$ which follows from (2), we obtain

$$
\mathbb{E} e^{-q \tau}=\mathbb{P}\left(\tau<e_{q}\right)=\mathbb{P}\left(\underline{Q}_{e_{q}}=0\right)=\frac{\bar{\kappa}(0,0)}{\bar{\kappa}(q, 0)}=\mathbb{E} e^{-q \bar{G}}
$$

showing that $\tau$ and $\bar{G}:=\bar{G}_{\infty}$, the (first) time of the overall maxima, have the same distribution (which was concluded, using another line of argumentation, in [3] as well).

\section{More refined quantities}

In this section we analyze a few more refined quantities that are related to the minimum workload. In this respect observe that

$$
\underline{G}_{e_{q}}=\sup \left\{t \in\left[0, e_{q}\right]: Q_{t} \wedge Q_{t-}=\underline{Q}_{e_{q}}\right\}
$$

is also the (last) time of the minimal workload, see Figure 1. In the sequel we find it convenient to distinguish between the following two cases: $\tau>e_{q}$ and $\tau<e_{q}$, i.e., if the residual busy period is still going on at time $e_{q}$, or not. In the second case we look at the first and the last times when the workload attains the value 0 , i.e. the time $\tau$ when the first busy period finishes and the time $\underline{G}_{e_{q}}$ when the last busy period starts. 


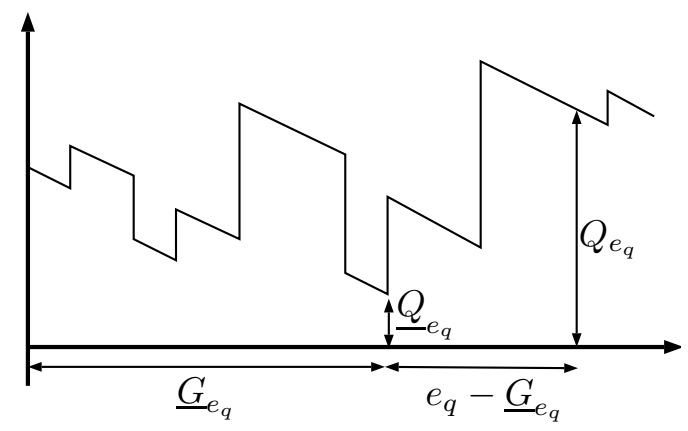

(a) Ongoing busy period: $\tau>e_{q}$

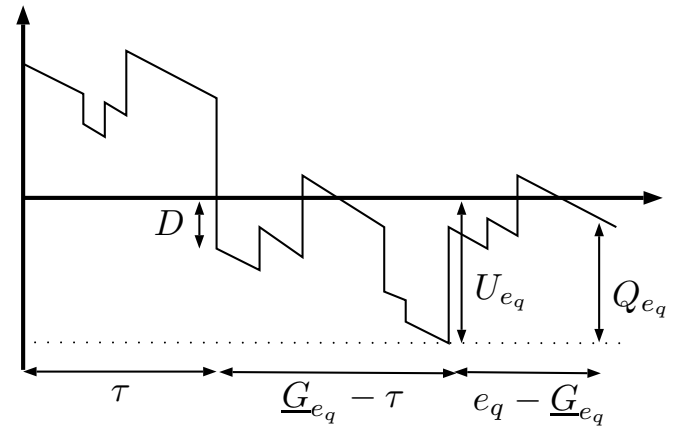

(b) Finished busy period: $\tau<e_{q}$

Figure 1: Schematic queueing process

\subsection{Ongoing busy period: $\tau>e_{q}$}

Note that (9) combined with (10) provides us with the equality

$$
\begin{aligned}
\mathbb{E}\left(e^{-\theta \underline{Q}_{e_{q}}} ; \tau>e_{q}\right) & =\mathbb{E} e^{-\theta \underline{Q}_{e_{q}}}-\mathbb{P}\left(\tau<e_{q}\right) \\
& =\frac{\bar{\kappa}(0,0) \bar{\kappa}(q, \theta)}{\bar{\kappa}(0, \theta)} \frac{\bar{\kappa}(0,0)}{\bar{\kappa}(q, 0)}=\frac{\bar{\kappa}(0,0)(\bar{\kappa}(q, \theta)-\bar{\kappa}(0, \theta))}{\bar{\kappa}(q, 0)},
\end{aligned}
$$

describing the distribution of $\underline{Q}_{e_{q}}$ on the event $\tau>e_{q}$. The following result gives a simple expression of the joint transform of various quantities related to the minimal workload in an ongoing busy period.

Proposition 2. For any $\theta, \alpha, \beta, \gamma, q \geq 0$,

$$
\mathbb{E}\left(e^{-\theta \underline{Q}_{e_{q}}-\alpha Q_{e_{q}}-\beta \underline{G}_{e_{q}}-\gamma\left(e_{q}-\underline{G}_{e_{q}}\right)} ; \tau>e_{q}\right)=\frac{q}{\beta+q} \frac{\bar{\kappa}(0,0)(\bar{\kappa}(q+\beta, \theta+\alpha)-\bar{\kappa}(0, \theta+\alpha))}{\bar{\kappa}(0, \theta+\alpha) \bar{\kappa}(q+\gamma, \alpha)} .
$$

Proof. By appealing to the usual splitting argument, we can write

$$
\begin{aligned}
\mathbb{E}\left(e^{-\theta \underline{Q}_{e_{q}}-\beta\left(e_{q}-\underline{G}_{e_{q}}\right)} ; \tau>e_{q}\right) & =\mathbb{E}\left(e^{-\theta \underline{Q}_{e_{q}}} ; \tau>e_{q}\right) \mathbb{E} e^{-\beta\left(e_{q}-\underline{G}_{e_{q}}\right)} \\
& =\mathbb{E}\left(e^{-\theta \underline{Q}_{e_{q}}} ; \tau>e_{q}\right) \frac{\bar{\kappa}(q, 0)}{\bar{\kappa}(q+\beta, 0)}=\frac{\bar{\kappa}(0,0)(\bar{\kappa}(q, \theta)-\bar{\kappa}(0, \theta))}{\bar{\kappa}(0, \theta) \bar{\kappa}(q+\beta, 0)},
\end{aligned}
$$

see (5) and (11). Now realize that

$$
\mathbb{E}\left(f\left(e_{q}\right) e^{-\beta e_{q}}\right)=\frac{q}{\beta+q} \mathbb{E} f\left(e_{q+\beta}\right)
$$

for any Borel function $f$. It therefore immediately follows that

$$
\mathbb{E}\left(e^{-\theta \underline{Q}_{e_{q}}-\beta\left(e_{q}-\underline{G}_{e_{q}}\right)} ; \tau>e_{q}\right)=\frac{q}{\beta+q} \mathbb{E}\left(e^{-\theta \underline{Q}_{e_{q+\beta}}+\beta T_{e_{q+\beta}}} ; \tau>e_{q+\beta}\right) .
$$

Replacing consistently $q+\beta$ by $q$, and $\beta$ by $-\beta$, we thus obtain

$$
\mathbb{E}\left(e^{-\theta \underline{Q}_{e_{q}}-\beta \underline{G}_{e_{q}}} ; \tau>e_{q}\right)=\frac{q}{\beta+q} \frac{\bar{\kappa}(0,0)(\bar{\kappa}(q+\beta, \theta)-\bar{\kappa}(0, \theta))}{\bar{\kappa}(0, \theta) \bar{\kappa}(q, 0)} .
$$


Finally, again relying on the splitting argument, we arrive at

$$
\begin{aligned}
& \mathbb{E}\left(e^{-\theta \underline{Q}_{e_{q}}-\alpha Q_{e_{q}}-\beta \underline{G}_{e_{q}}-\gamma\left(e_{q}-\underline{G}_{e_{q}}\right)} ; \tau>e_{q}\right) \\
& =\mathbb{E}\left(e^{-(\alpha+\theta) \underline{Q}_{e_{q}}-\beta \underline{G}_{e_{q}}} ; \tau>e_{q}\right) \mathbb{E}\left(e^{-\alpha\left(X_{e_{q}}-\underline{X}_{e_{q}}\right)-\gamma\left(e_{q}-\underline{G}_{e_{q}}\right)}\right) \\
& =\frac{q}{\beta+q} \frac{\bar{\kappa}(0,0)(\bar{\kappa}(q+\beta, \theta+\alpha)-\bar{\kappa}(0, \theta+\alpha))}{\bar{\kappa}(0, \theta+\alpha) \bar{\kappa}(q, 0)} \frac{\bar{\kappa}(q, 0)}{\bar{\kappa}(q+\gamma, \alpha)},
\end{aligned}
$$

see (5), completing the proof.

4.2 Finished busy period: $\tau<e_{q}$

In the case $\tau<e_{q}$ we define two random quantities:

$$
U_{e_{q}}:=-\left(Q_{0}+\underline{X}_{e_{q}}\right), \quad D:=-\left(Q_{0}+X_{\tau}\right),
$$

see Figure 1(b). The first can be interpreted as the unused capacity, and the second as the sudden unused capacity at the end of the initial busy period. The latter quantity may be of limited interest, but it can be included in the joint transform without any additional work. First we start with a basic result complementing (11).

Proposition 3. For any $\theta, q \geq 0$,

$$
\mathbb{E}\left(e^{-\theta U_{e_{q}}} ; \tau<e_{q}\right)=\frac{\bar{\kappa}(0,0)}{\bar{\kappa}(q, 0)} \frac{\underline{\kappa}(q, \theta)-\underline{\kappa}(0, \theta)}{\underline{\kappa}(q, \theta)} .
$$

Proof. First we compute for $\theta=i \mathbb{R}$

$$
\begin{aligned}
\mathbb{E}\left(e^{-\theta U_{e_{q}}} ; \tau<e_{q}\right) & =\mathbb{E} e^{\theta\left(Q_{0}+\underline{X}_{e_{q}}\right)}-\mathbb{E}\left(e^{\theta \underline{Q}_{e_{q}}} ; \tau>e_{q}\right) \\
& =\frac{\bar{\kappa}(0,0)}{\bar{\kappa}(0,-\theta)} \frac{\underline{\kappa}(q, 0)}{\bar{\kappa}(q, \theta)}-\frac{\bar{\kappa}(0,0)(\bar{\kappa}(q,-\theta)-\bar{\kappa}(0,-\theta))}{\bar{\kappa}(0,-\theta) \bar{\kappa}(q, 0)},
\end{aligned}
$$

see (5), (4) and (11). Using (3) this can be rewritten as

$$
\frac{\bar{\kappa}(0,0)}{\bar{\kappa}(q, 0) \bar{\kappa}(0,-\theta)}\left(\frac{q}{\underline{\kappa}(q, \theta)}-\bar{\kappa}(q,-\theta)+\bar{\kappa}(0,-\theta)\right) .
$$

Finally, using (6) we can express $\bar{\kappa}(q,-\theta)=(q-\psi(-i \theta)) / \underline{\kappa}(0, \theta)$. Plugging this in yields that the expression in the previous display equals

$$
\frac{\bar{\kappa}(0,0) \underline{\kappa}(0, \theta)}{-\bar{\kappa}(q, 0) \psi(-i \theta)}\left(\frac{\psi(-\mathrm{i} \theta)}{\underline{\kappa}(q, \theta)}-\frac{\psi(-i \theta)}{\underline{\kappa}(0, \theta)}\right),
$$

which easily reduces to the expression in the statement. Finally, analytic continuation shows that it is true for all $\theta \in \mathbb{C}$ with $\Re(\theta) \geq 0$.

Proposition 4. For any $\theta, \alpha, \beta, \gamma, u, v, q \geq 0$,

$$
\begin{gathered}
\mathbb{E}\left(e^{-\alpha D-\beta U_{e_{q}}-\gamma Q_{e_{q}}-u \tau-v\left(\underline{G}_{e_{q}}-\tau\right)-w\left(e_{q}-\underline{G}_{e_{q}}\right)} ; \tau<e_{q}\right) \\
=\frac{q}{q+u} \frac{\bar{\kappa}(0,0)(\underline{\kappa}(q+u, \alpha+\beta)-\underline{\kappa}(0, \alpha+\beta))}{\bar{\kappa}(q+w, \gamma) \underline{\kappa}(q+v, \beta)} .
\end{gathered}
$$


Proof. By splitting we have

$$
\mathbb{E}\left(e^{-\alpha U_{e_{q}}-u\left(e_{q}-\underline{G}_{e_{q}}\right)} ; \tau<e_{q}\right)=\mathbb{E}\left(e^{-\alpha U_{e_{q}}} ; \tau<e_{q}\right) \mathbb{E} e^{-u\left(e_{q}-\underline{G}_{e_{q}}\right)}=\frac{\bar{\kappa}(0,0)(\underline{\kappa}(q, \alpha)-\underline{\kappa}(0, \alpha))}{\underline{\kappa}(q, \alpha) \bar{\kappa}(q+u, 0)},
$$

see also (5) and Proposition 3. Moreover, using (12) we write

$$
\mathbb{E}\left(e^{-\alpha U_{e_{q}}-u\left(e_{q}-\underline{G}_{e_{q}}\right)} ; \tau<e_{q}\right)=\frac{q}{q+u} \mathbb{E}\left(e^{-\alpha U_{e_{q+u}}+u T_{e_{q+u}}} ; \tau<e_{q+u}\right) .
$$

As before, changing parameters leads to

$$
\begin{aligned}
\mathbb{E}\left(e^{-\alpha U_{e_{q}}-u \underline{G}_{e_{q}}} ; \tau<e_{q}\right) & =\frac{q}{q+u} \mathbb{E}\left(e^{-\alpha U_{e_{q+u}}+u\left(e_{q+u}-T_{e_{q+u}}\right)} ; \tau<e_{q+u}\right) \\
& =\frac{q}{q+u} \frac{\bar{\kappa}(0,0)(\underline{\kappa}(q+u, \alpha)-\underline{\kappa}(0, \alpha))}{\underline{\kappa}(q+u, \alpha) \bar{\kappa}(q, 0)} .
\end{aligned}
$$

Next, we apply the strong Markov property at the time $\tau$ to obtain the identity

$$
\mathbb{E}\left(e^{-\alpha U_{e_{q}}-u \underline{G}_{e_{q}}} ; \tau<e_{q}\right)=\mathbb{E}\left(e^{-\alpha D-u \tau} ; \tau<e_{q}\right) \mathbb{E}\left(e^{\alpha \underline{X}_{e_{q}}-u \underline{G}_{e_{q}}}\right),
$$

which leads to

$$
\mathbb{E}\left(e^{-\alpha D-u \tau} ; \tau<e_{q}\right)=\frac{\bar{\kappa}(0,0)(\underline{\kappa}(q+u, \alpha)-\underline{\kappa}(0, \alpha))}{q+u}
$$

using (4) and (2). Finally, we write using the strong Markov property at $\tau$ and splitting at $\underline{G}_{e_{q}}$

$$
\begin{aligned}
& \mathbb{E}\left(e^{-\alpha D-\beta U_{e_{q}}-\gamma Q_{e_{q}}-u \tau-v\left(\underline{G}_{e_{q}}-\tau\right)-w\left(e_{q}-\underline{G}_{e_{q}}\right)} ; \tau<e_{q}\right) \\
& =\mathbb{E}\left(e^{-(\alpha+\beta) D-u \tau} ; \tau<e_{q}\right) \mathbb{E} e^{\beta \underline{X}_{e_{q}}-v \underline{G}_{e_{q}}} \mathbb{E} e^{-\gamma\left(X_{e_{q}}-\underline{X}_{e_{q}}\right)-w\left(e_{q}-\underline{G}_{e_{q}}\right)} \\
& =\frac{\bar{\kappa}(0,0)(\underline{\kappa}(q+u, \alpha+\beta)-\underline{\kappa}(0, \alpha+\beta))}{q+u} \frac{\underline{\kappa}(q, 0)}{\underline{\kappa}(q+v, \beta)} \frac{\bar{\kappa}(q, 0)}{\bar{\kappa}(q+w, \gamma)},
\end{aligned}
$$

which in view of (3) completes the proof.

\section{Minimal workload in a queue conditioned to stay positive}

In this section we focus on the law of $\underline{Q}_{e_{q}}$ conditional on the queue not having idled between 0 and $e_{q}$, i.e., $\tau>e_{q}$, and provide an alternative representation of this law in the limiting case when $q \downarrow 0$. We also comment on the relation of this limit law to Lévy processes conditioned to stay positive. It follows directly from (10) and (11) that

$$
\mathbb{E}\left(e^{-\theta \underline{Q}_{e_{q}}} \mid \tau>e_{q}\right)=\mathbb{E}\left(e^{-\theta \underline{Q}_{e_{q}}} ; \tau>e_{q}\right) / \mathbb{P}\left(\tau>e_{q}\right)=\frac{\bar{\kappa}(0,0)}{\bar{\kappa}(0, \theta)} \frac{\bar{\kappa}(q, \theta)-\bar{\kappa}(0, \theta)}{\bar{\kappa}(q, 0)-\bar{\kappa}(0,0)} .
$$

Note, however, that the corresponding conditional law does not have a direct link (via Laplace transform) to its transient counterpart, i.e. when $e_{q}$ is replaced by $t$.

In the following we assume that $\bar{\kappa}^{\prime}(0,0)<\infty$ (and so also $\bar{\kappa}^{\prime}(0, \theta)<\infty$ for $\theta \geq 0$ ), where the derivative of $\bar{\kappa}(q, \theta)$ is taken with respect to $q$. According to (10) this requirement is equivalent to $\mathbb{E} \tau=\mathbb{E} \bar{G}<\infty$; see Proposition 6 for an example when this assumption does not hold. Now it follows from (13) that

$$
\lim _{q \downarrow 0} \mathbb{E}\left(e^{-\theta \underline{Q}_{e_{q}}} \mid \tau>e_{q}\right)=\frac{\bar{\kappa}(0,0)}{\bar{\kappa}(0, \theta)} \frac{\bar{\kappa}^{\prime}(0, \theta)}{\bar{\kappa}^{\prime}(0,0)}=\frac{\log (\bar{\kappa}(0, \theta))^{\prime}}{\log (\bar{\kappa}(0,0))^{\prime}} .
$$

Along the same lines, one can establish the generalization

$$
\lim _{q \downarrow 0} \mathbb{E}\left(e^{-\theta \underline{Q}_{e_{q}}-\alpha Q_{e_{q}}} \mid \tau>e_{q}\right)=\frac{\bar{\kappa}(0,0)}{\bar{\kappa}(0, \alpha)} \frac{\log (\bar{\kappa}(0, \theta+\alpha))^{\prime}}{\log (\bar{\kappa}(0,0))^{\prime}} .
$$


Remark 2. It must be noted that the limit law of $\underline{Q}_{e_{q}} \mid \tau>e_{q}$ as $q \downarrow 0$ does not coincide with the so-called quasi-stationary distribution of the minimal workload, i.e. with that of $\underline{Q}_{t} \mid \tau>t$ as $t \rightarrow \infty$, even though $e_{q} \rightarrow \infty$ a.s. as $q \downarrow 0$. Roughly speaking, conditioning on $\left\{\tau>e_{q}\right\}$ does not only force $\tau$ to be large, but also makes $e_{q}$ to appear smaller. In this respect it may be helpful to mention that

$$
\lim _{q \downarrow 0} \mathbb{E}\left(e^{-\theta e_{q}} \mid \tau>e_{q}\right)=\frac{1-\mathbb{E} e^{-\theta \tau}}{\theta \mathbb{E} \tau},
$$

i.e. the limit law of $e_{q} \mid \tau>e_{q}$ is a proper distribution, the residual life distribution associated to $\tau$. For related results on the quasi-stationary behaviour of reflected one-sided Lévy processes we refer to e.g. [7].

Remark 3. Importantly, one way to construct a Lévy process started in $x$ and conditioned to stay positive (in the usual sense) is to condition on $\left\{\tau>e_{q}\right\}$ and then let $q \downarrow 0$, see [2, Prop. 1]. The distribution of the infimum of this process is characterized in [2, Thm. 1]. Note, however, that the limit distribution of $\underline{Q}_{e_{q}} \mid \tau>e_{q}$ can not be obtained from this result by integrating with respect to $\mathbb{P}\left(Q_{0} \in \mathrm{d} x\right)$. The main reason is that

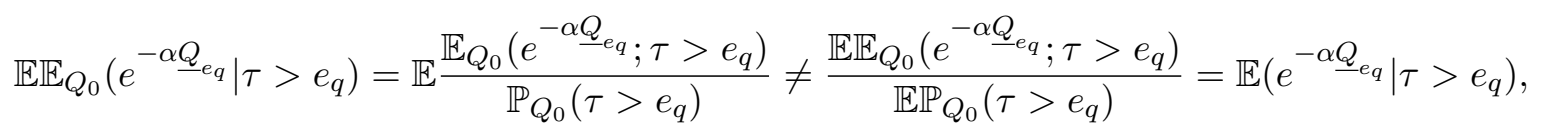

because the event upon which we condition depends on $Q_{0}$.

Proposition 5. Assume that $\bar{\kappa}^{\prime}(0,0)<\infty$. Then the limit laws of

$$
\underline{Q}_{e_{q}} \mid \tau>e_{q}, \quad \text { and } \quad X_{e_{q}} \mid X_{e_{q}}>0
$$

coincide as $q \downarrow 0$.

Proof. Using (2) observe that

$$
q \log (\bar{\kappa}(q, \theta))^{\prime}=\int_{0}^{\infty} \int_{0}^{\infty} q e^{-q t-\theta x} \mathbb{P}\left(X_{t} \in \mathrm{d} x\right) \mathrm{d} t=\mathbb{E}\left(e^{-\theta X_{e_{q}}} ; X_{e_{q}}>0\right) .
$$

Hence

$$
\frac{\log (\bar{\kappa}(0, \theta))^{\prime}}{\log (\bar{\kappa}(0,0))^{\prime}}=\lim _{q \downarrow 0} \frac{\log (\bar{\kappa}(q, \theta))^{\prime}}{\log (\bar{\kappa}(q, 0))^{\prime}}=\lim _{q \downarrow 0} \frac{\mathbb{E}\left(e^{\left.-\theta X_{e_{q}} ; X_{e_{q}}>0\right)}\right.}{\mathbb{P}\left(X_{e_{q}}>0\right)}=\lim _{q \downarrow 0} \mathbb{E}\left(e^{-\theta X_{e_{q}}} \mid X_{e_{q}}>0\right) .
$$

The proof is complete in view of (14).

This result has a simple intuitive explanation. Firstly, on the left hand side we have the limit of $Q_{0}+\underline{X}_{e_{q}} \mid Q_{0}+\underline{X}_{e_{q}}>0$. Secondly, it holds that $X_{e_{q}}=\left(X_{e_{q}}-\underline{X}_{e_{q}}\right)+\underline{X}_{e_{q}}$, where the two terms on the right are independent and the distribution of the first converges to that of $Q_{0}$ as $q \downarrow 0$.

Let us conclude by giving yet another representation of the limit law of $\underline{Q}_{e_{q}} \mid \tau>e_{q}$ in the spectrally one-sided cases.

Proposition 6. Assume that $X$ is either spectrally positive or spectrally negative. Then the limiting law of $\underline{Q}_{e_{q}} \mid \tau>e_{q}$ as $q \downarrow 0$ is the residual life distribution associated to $Q_{0}$, i.e.

$$
\lim _{q \downarrow 0} \mathbb{E}\left(e^{-\theta \underline{Q}_{e_{q}}} \mid \tau>e_{q}\right)=\frac{1-\mathbb{E} e^{-\theta Q_{0}}}{\theta \mathbb{E} Q_{0}} .
$$

Moreover, $\bar{\kappa}^{\prime}(0,0)=\infty$ if and only if $X$ is a spectrally positive process with $\operatorname{var}\left(X_{1}\right)=\infty$, in which case $\mathbb{E} Q_{0}=\infty$ and $\underline{Q}_{e_{q}} \mid \tau>e_{q}$ converges to $\infty$ as $q \downarrow 0$. 
Proof. The identity (15) is easily verified using (14) and the explicit expressions for $\bar{\kappa}(\cdot, \cdot)$ in both cases, see, e.g., [6, Sec. 6.5.2]. These explicit expressions also show that in the spectrally negative case we must have $\bar{\kappa}^{\prime}(0,0)<\infty$, whereas in the spectrally positive case $\bar{\kappa}^{\prime}(0,0)<\infty$ iff $\phi^{\prime \prime}(0)=\infty$, where $\phi(\theta)=\log \mathbb{E} e^{-\theta X_{1}}$. The latter is equivalent to $\operatorname{var}\left(X_{1}\right)=\infty$ and implies that (15) results in 0 .

It seems unlikely that (15) holds in general. It would be interesting to characterize all the Lévy processes drifting to $-\infty$ for which (15) is true. Another challenging problem is to express the joint transform $\mathbb{E} e^{-\alpha Q_{0}-\beta Q_{e_{q}}}$ (or, closely related, the joint transform $\mathbb{E} e^{-\alpha Q_{0}-\beta \underline{Q}_{e_{q}}}$ ) through the functions $\bar{\kappa}(\cdot, \cdot)$ and $\underline{\kappa}(\cdot, \cdot)$.

\section{References}

[1] J. Bertoin (1998). Lévy processes (Vol. 121). Cambridge university press.

[2] L. Chaumont and R. Doney (2005). On Lévy processes conditioned to stay positive. Electron. J. Probab. 10(28), pp. 948-961.

[3] K. DȩBicki, T. Dieker, and T. Rolski (2007). Quasi-product forms for Lévy-driven fluid networks. Math. Oper. Res. 32, pp. 629-647.

[4] K. DȨBicki, K. Kosiński, and M. MANDJes (2012). On the infimum attained by a reflected Lévy process. Queueing Syst. 70, pp. 23-35.

[5] K. DȩBicki and M. Mandjes (2015). Queues and Lévy Fluctuation Theory. Springer, Berlin, Germany.

[6] A. Kyprianou (2006). Introductory Lectures on Fluctuations of Lévy Processes with Applications. Springer, Berlin, Germany.

[7] M. Mandjes, Z. Palmowski, and T. Rolski (2012). Quasi-stationary workload of a Lévy-driven queue. Stoch. Mod. 28, pp. 413-432. 